\title{
trans-10,cis-12 conjugated linoleic acid alters lipid metabolism of goat mammary epithelial cells by regulation of de novo synthesis and the AMPK signaling pathway
}

\author{
T. Y. Zhang, J. T. Huang, H. B. Tian, Y. Ma, Z. Chen, J. J. Wang, H. P. Shi, ${ }^{1}$ and J. Luo ${ }^{1}$ \\ Shaanxi Key Laboratory of Molecular Biology for Agriculture, College of Animal Science and Technology, Northwest A\&F University, Yangling, \\ Shaanxi, P. R. China 712100
}

\begin{abstract}
The trans-10,cis-12 isomer of conjugated linoleic acid (t10c12-CLA) is a biohydrogenation intermediate in the rumen and has been shown to cause milk fat depression in dairy goats. However, few studies have focused on the in vitro molecular mechanisms involved in the response of the goat mammary gland to t10c12CLA. In the present study, RNA sequencing technology was used to investigate the effects of t10c12-CLA on goat mammary epithelial cells. From the data, 25,153 annotated transcripts were obtained, and differentially expressed genes were selected based on a false discovery rate $<0.05$. Candidate genes and potent cellular signaling pathways were identified through Gene Ontology (GO) and pathway analysis. Next, real-time quantitative PCR and Western blot analyses were used to verify the results of the RNA sequencing data. The results indicated that t10c12-CLA inhibits fatty acid synthesis through downregulation of genes involved in de novo fatty acid synthesis, and this process is likely correlated with the activation of the AMP-activated protein kinase signaling pathways.
\end{abstract}

Key words: trans-10,cis-12 conjugated linoleic acid, mammary, RNA sequencing, AMP-activated protein kinase (AMPK)

\section{INTRODUCTION}

The mammalian body can adjust its metabolism in response to a variety of nutritional conditions, and the regulation of gene expression and altered enzyme activities in metabolic pathways play critical roles in this adaptive response (Nakamura et al., 2004). Milk con-

\footnotetext{
Received March 2, 2017.

Accepted January 22, 2018.

${ }^{1}$ Corresponding authors: huaipingshi@nwsuaf.edu.cn and luojun@ nwsuaf.edu.cn
}

tent and composition can be affected by diet (Peterson et al., 2004), and significant changes occur in milk FA composition in response to altered feeding conditions, especially in dairy cattle and goat (Chilliard et al., 2007). The trans-10, cis-12 isomer of conjugated linoleic acid (t10c12-CLA), a biohydrogenation intermediate in the rumen, has been known to decrease milk fat synthesis (Kadegowda et al., 2010; Hussein et al., 2013; Harvatine et al., 2014). In dairy cattle, t10c12-CLA is a primary factor causing milk fat depression (Baumgard et al., 2000, 2002).

Studies in bovine MAC-T cells indicated that inhibition of de novo fatty acid synthesis by t10c12-CLA (100 $\mu M)$ may be due to reduced proteolytic activation of sterol regulatory element-binding transcription factor 1 (SREBF-1), downregulation of thyroid hormone responsive (THRSP), and changes to a series of lipogenic genes (Peterson et al., 2004; Harvatine and Bauman, 2006). Gene expression data associated with fatty acid uptake, intracellular activation, intracellular transport, de novo synthesis, and esterification after treatment with t10c12-CLA indicate negative effects on lipogenic gene networks in bovine mammary cells (Kadegowda et al., 2009). In vivo investigations using the microarray method in murine mammary tissue indicated that the effects of t10c12-CLA might encompass inhibition of peroxisome proliferator-activated receptor gamma (PPAR $\gamma)$ signaling and fatty acid biosynthesis, as well as induction of inflammation and endoplasmic reticulum stress (Kadegowda et al., 2013).

However, few studies have focused on the molecular mechanisms underlying those responses in the goat mammary gland. Although previous murine and bovine studies provide guidance for similar studies in dairy goat, direct application of those results are limited. First, those reports may not directly represent the effects of t10c12-CLA in dairy goat due to species-specific differences. Second, methods such as real-time quantitative PCR (RT-qPCR) and microarray might not detect the global dynamic range of gene expression in response to t10c12-CLA. RNA is a dynamic and diverse biomol- 
ecule, playing an essential role in numerous biological processes (Byron et al., 2016). Thus, capturing whole transcriptome information is critical in a comprehensive investigation of the response to t10c12-CLA in the goat mammary gland. RNA sequencing provides an in-depth view of the transcriptome, allowing study of global gene expression changes and alternative splicing in any cell population, overcoming limits of microarray assays that are unable to detect novel changes or identify novel isoforms of known genes (Mortazavi et al., 2008; Jakhesara et al., 2013). In the present study, RNA sequencing technology was applied to evaluate the effects of t10c12-CLA on goat mammary epithelial cells (GMEC). Important genes and potential cellular signaling pathways involved in the response were identified and verified by RT-qPCR and Western blot.

\section{MATERIALS AND METHODS}

\section{Preparation of t10c12-CLA}

A stock solution of $30 \mathrm{~m} M$ t10c12-CLA (Sigma-Aldrich, St, Louis, MO) was dissolved in $95 \%$ ethanol and $30 \mathrm{~m} M \mathrm{NaOH}$ as previously described (Kadegowda et al., 2009). A solution of $95 \%$ ethanol and $30 \mathrm{mM} \mathrm{NaOH}$ served as the control.

\section{Cell Culture and Treatments}

The experiment was conducted under the approval of the Institutional Animal Use and Care Committee of Northwest A\&F University (Shaanxi, China). The GMEC were isolated from Xinong Saanen goats during peak lactation and cultured in a basal Dulbecco's modified Eagle medium/F12 (DMEM/F12) medium (Hyclone, South Logan, UT) containing $1 \mu \mathrm{g} / \mathrm{mL}$ hydrocortisone (Sigma-Aldrich), $5 \mu \mathrm{g} / \mathrm{mL}$ insulin, 100 $\mathrm{U} / \mathrm{mL}$ penicillin, $100 \mathrm{mg} / \mathrm{mL}$ streptomycin (Harbin Pharmaceutical Group, Harbin, China), $5 \mathrm{~m} M$ sodium acetate, $10 \mathrm{ng} / \mathrm{mL}$ epidermal growth factor 1 (Invitrogen Corp., Frederick, MD), and 10\% fetal bovine serum (Gibco/Thermo Fisher Scientific, Waltham, MA) at $37^{\circ} \mathrm{C}$ in a humidified atmosphere with $5 \% \mathrm{CO}_{2}$. The serum-free DMEM/F12 medium contained no serum, 1 $\mathrm{mg} / \mathrm{mL}$ BSA (Sigma-Aldrich), and $2 \mu \mathrm{g} / \mathrm{mL}$ prolactin (Sigma-Aldrich). For the experimental treatment of GMEC, the basal medium was changed to serum-free medium containing CLA or the control. The GMEC were grown in 6-well culture plates (for RNA extraction) or $60-\mathrm{mm}$ culture plates (for Western blot) until approximately $80 \%$ confluence, and were then treated with serum-free medium containing 0 (control; CTR), 100 (treatment TR1), or 200 (treatment TR2) $\mu M$ t10c12-CLA for $12 \mathrm{~h}$.

\section{RNA Extraction, Library Construction, and Sequencing}

For RNA sample preparation, each treatment had 8 replicates. The total RNA of each group was isolated using the Trizol reagent according to the manufacturer's instructions (Invitrogen, Carlsbad, CA). The extracted samples were separated on $1 \%$ agarose gels to detect RNA degradation and contamination. The RNA concentration was measured using the Qubit RNA Assay Kit in Qubit2.0 Fluorometer (Life Technologies). The purity and integrity of RNA was detected using the Nano 6000 Assay kit of the Bioanalyzer Agilent 2100 system (Agilent Technologies, Palo Alto, CA). Finally, 5 samples of homogenized total RNAs with RNA integrity number values $>8$ for each treatment were mixed to make one sample at the same concentration. Three sequencing libraries were prepared: control group (CTR), $100 \mu M$ t10c12-CLA (TR1), and $200 \mu M$ t10c12-CLA (TR2), using the IlluminaTruSeq RNA Sample Preparation kit (Illumina, San Diego, CA) according to the manufacturer's instructions and adding 4 index codes to attribute sequence to each sample. After library construction, the Qubit2.0 system was used to measure the concentration of cDNA libraries. The libraries were then diluted to $1 \mathrm{ng} / \mu \mathrm{L}$, and the insert size was measured using the Agilent 2100 system. Then, the cDNA libraries were quantified by RT-qPCR (library effective concentration $>2 \mathrm{n} M$ ) to ensure the quality. The clustering of the index-coded samples was performed on a cBot Cluster Generation System using the TruSeq PE Cluster Kit v3-cBotHS (Illumina) according to the manufacturer's instructions. The library preparations were sequenced on an Illumina HiSeq 2500 platform and 150-bp paired-end reads were generated. The sequenced RNA-seq data for CTR, TR1, and TR2 are available from National Center for Biotechnology Information Sequences Read Archive with accession numbers SRR5714864, SRR5714865, and SRR5714866.

\section{Mapping and Assembly of Sequence Reads}

The raw data (in fastq format) were first processed using in-house Perl scripts, and then clean data were obtained by removal of adapter sequences, reads containing poly-N $(>10 \%)$, and low quality reads (more than $50 \%$ bases in which phred quality score $\leq 5$ in a read) from the raw data. The indices of clean bases, error rate, Q20 (proportion of bases with a phred base quality score $>20$ ), Q30 (proportion of bases with a phred base quality score $>30$ ), and GC content from the sequence reads were assessed to ensure high quality of the clean data. Using the Capra hircus (goat) genome (ID: 10731) as reference genome, an index of 
the reference genome was built using Bowtie v0.12.8 (Langmead and Salzberg, 2012). Next, the paired-end clean data were aligned to the reference genome using TopHat v2.0.7 software (Trapnell et al., 2009).

\section{Quantification and Differential Expression Analysis of Transcripts}

Before performing the differential gene expression analysis for each library, the read counts were adjusted using the edgeR program package through one scaling normalized factor (Robinson, 2010). Differential expression analysis of 2 conditions (CTR vs. TR1, CTR vs. TR2, and TR1 vs. TR2) was performed using the DEGeq R package (Robinson and Oshlack, 2010; Wang et al., 2010). The $P$-values were adjusted using the Benjamini and Hochberg (1995) method to control the false discovery rate. The corrected $P$-value of 0.05 was set as the threshold for significantly differential expression. Venn diagrams were used to illustrate the number of differentially expressed genes (DEG) shared between the comparison groups and the DEG particular to individual groups.

\section{Enrichment Analysis of Differentially Expressed Transcripts}

Gene Ontology (GO) is a major bioinformatics initiative to unify the representation of gene and gene product attributes across all species (http:// geneontology.org/). Using the GOseq $\mathrm{R}$ package based on the Wallenius non-central hyper-geometric distribution, GO enrichment analysis was performed (Young et al., 2010). The Kyoto Encyclopedia of Genes and Genomes (KEGG) is a database resource to understand high-level functions and utilities of the biological system, such as the cell, the organism, and the ecosystem, from molecular-level information, especially large-scale molecular data sets generated by genome sequencing and other high-throughput experimental technologies (http://www.genome.jp/kegg/). Using KOBAS (2.0), we performed a KEGG enrichment analysis (Xie et al., 2011). To show the number of up- and downregulated genes, we used a bar chart to present the KEGG data, as in Sajnani et al. (2012).

\section{$R T-q P C R$}

The first-strand cDNA was synthesized from $1 \mu \mathrm{g}$ of purified total RNA from each treatment using the PrimeScript RT reagent Kit with gDNA Eraser (Takara, Tokyo, Japan) according to the manufacturer's instructions. The RT-qPCR was performed according to the manufacturer's instructions using SYBR green (SYBR Premix Ex Taq II, Perfect Real Time, Takara).
Ubiquitously expressed transcript $(U X T)$, ribosomal protein S9 (RPS9), and mitochondrial ribosomal protein L39 (MRPL39) were used as internal control genes. The stability of the internal control genes and the reliability of the calculated normalization factor were evaluated using geNorm software. The M-values (expression stability) were $0.475,0.552$ and 0.558 for UXT, RPS9, and MRPL39, and the V-value (pairwise variation) was 0.107 ; thus, these internal control genes were stable according to the thresholds (M-value $\leq 1.5$ and $\mathrm{V}$-value $\leq 0.15$ ) suggested by Vandesompele et al. (2002). All of the qPCR reactions were performed in a Bio-Rad CFX96 sequence detector (Bio-Rad Laboratories Inc., Hercules, CA), and the data were normalized to the geometric mean of 3 internal controls. Data were then analyzed using the relative quantification $\left(2^{-\Delta \Delta} \mathrm{Ct}\right)$ cycle threshold method. Primer sequences are shown in Supplemental Table S1 (https://doi.org/10 $.3168 /$ jds.2017-12822).

\section{Western Blot}

For Western blot, total cellular protein was harvested and lysed with RIPA (radioimmunoprecipitation assay) buffer (Solarbio, Beijing, China) containing protease inhibitor cocktail (Roche, Mannheim, Germany). The cell lysate was extracted and then boiled with Lane Marker Loading Buffer (CWBIO, Beijing, China) according to the manufacturer's instructions. Western blot was conducted according to a method previously described (Xu et al., 2016). In brief, the total protein of each treatment was separated by SDS-PAGE and the blot was transferred to nitrocellulose membrane (Pall Corporation, New York, NY) by trans-blot SD semi-dry transfer cell (Bio-Rad Laboratories). The membrane was then blocked using 5\% skim milk before incubation with primary antibodies. Polyclonal rabbit anti-FASN, anti-SCD (1:300 dilution, prepared in our laboratory), and phosphorylated (p)-ACACA (acetylCoA carboxylase 1) Ser9 (1:1,000 dilution, Cell Signaling), and monoclonal rabbit-anti-p-AMPK $\alpha$ Thr172 (1:1,000 dilution, Cell Signaling), AMPK $\alpha$ (1:1,000 dilution, Cell Signaling), and mouse anti-ACTB (1:4,000 dilution, CWBIO) were used as the primary antibodies. Polyclonal goat anti-mouse IgG coupled to horseradish peroxidase (HRP, 1:4,000 dilution, CWBIO) and polyclonal goat anti-rabbit IgG coupled to HRP (1:4,000 dilution, CWBIO) were used as secondary antibodies. Signals were detected using the chemiluminescent ECL Western blot detection system (Bio-Rad Laboratories). The intensities of bands were quantified by densitometry using ImageJ software (National Institutes of Health, Bethesda, MD), and were normalized to nonphosphorylated kinase or $\beta$-actin. The values were 
Table 1. Overview of sequencing reads ${ }^{1}$ with different CLA treatments in goat mammary epithelial cells (GMEC)

\begin{tabular}{|c|c|c|c|c|c|c|c|}
\hline Treatment $^{2}$ & Raw reads & Clean reads & Clean bases & Error rate $(\%)$ & Q20 (\%) & Q30 (\%) & GC content $(\%)$ \\
\hline TR1 & $27,753,332$ & $26,886,038$ & $3.36 \mathrm{G}$ & 0.03 & 96.32 & 92.59 & 52.15 \\
\hline TR2 & $25,431,708$ & $24,777,838$ & $3.1 \mathrm{G}$ & 0.03 & 96.26 & 92.5 & 52.04 \\
\hline
\end{tabular}

${ }^{1} \mathrm{Q} 20=$ proportion of bases with a phred base quality score greater than 20; that is, the proportion of read bases whose error rate is less than $1 \%$. Q30 = proportion of bases with a phred base quality score greater than 30; that is, the proportion of read bases whose error rate is less than $0.1 \%$.

${ }^{2} \mathrm{CTR}=$ control $($ GMEC treated with $0 \mu M$ trans-10,cis-12 CLA); TR1 = GMEC treated with $100 \mu M$ trans-10,cis-12 CLA; TR2 = GMEC treated with $200 \mu M$ trans-10, cis-12 CLA).

expressed as the fold change versus a control protein band labeled as " $1.00 "$ in the figure.

\section{Statistical Analysis}

The fold change and significance of RNA-seq data were analyzed using the DEGeq R package (Robinson and Oshlack, 2010; Wang et al., 2010), as described in Materials and Methods: Quantification and Differential Expression Analysis of Transcripts, and a false discovery rate $<0.05$ was set as the threshold for significance. Experiments using RT-qPCR included 5 biological replicates. Western blot was performed using 3 biological replicates and a representative result was shown. The results are expressed as the means \pm standard errors for means. The RT-qPCR and Western blot quantification data were analyzed by one-way ANOVA using the general linear model procedure of SPSS 19.0 software (SPSS Inc., Chicago, IL) and Duncan's new multiple range test (Somasundaram et al., 2015). The correlations between the mRNA expression level from qPCR and RNA-seq for 9 DEG were estimated using the linear model of SPSS 19.0 software, as mentioned by Wang et al. (2015). Significance was declared at $P$ $<0.05$.

\section{RESULTS}

\section{Identification of Expressed Transcripts in GMEC in Response to CLA}

In this study, 28,257,738, 27,753,332, and 25,431,708 raw reads were generated for CTR, TR1, and TR2 treat- ments, respectively. After quality control, 2,728,064, $26,886,038$, and $24,777,838$ clean reads were obtained for the 3 groups, respectively, with quality values Q20 and Q30 at 96.28 and $92.52 \%$, respectively, as shown in Table 1. Using the goat genome as the reference, approximately $78 \%$ of the total reads were mapped. The uniquely mapped reads of the CTR, TR1, and TR2 groups corresponded to $76.8,76.23$, and $76.53 \%$ of the total, respectively, as shown in Supplemental Table S2 (https://doi.org/10.3168/jds.2017-12822).

The gene expression level was calculated according to the reads per kilobases per million reads (RPKM) method; considering RPKM >0.01 as "expressed," we identified 17,559, 17,455, and 17,982 expressed genes, respectively, from 25,153 annotated transcripts, which came from the 3 groups. To better categorize these expressed genes, the expression levels were divided into 3 groups: low (0.01 to 10 RPKM), medium (10 to 500 RPKM), and high ( $\geq 500$ RPKM), as shown in Table 2. The 20 most highly expressed genes of each group are presented in Figure 1.

\section{Identification of DEG and Functional Characterization in GMEC in Response to CLA}

The DEG are presented as TR1 versus CTR, TR2 versus CTR, and TR1 versus TR2 (Figure 2). Using the corrected $P$-value $<0.05$ as the threshold, there were 49, 854, and 623 DEG in TR1 versus CTR, TR2 versus CTR, and TR1 versus TR2, respectively (Supplemental Table S3; https://doi.org/10.3168/jds.2017-12822). To

Table 2. RNA sequencing gene expression results in goat mammary epithelial cells (GMEC) under different CLA treatments

\begin{tabular}{lccc}
\hline & \multicolumn{3}{c}{ CLA treatment $^{2}$} \\
\cline { 2 - 4 } RPKM interval $^{1}$ & $\mathrm{CTR}$ & $\mathrm{TR} 1$ & TR2 \\
\hline Expressed genes $(\mathrm{RPKM}>0.01)_{\text {Low expressed }(0.01-10)}$ & 17,559 & 17,455 & 17,982 \\
Medium expressed $(10-500)$ & $10,806(61.54 \%)$ & $10,634(60.92 \%)$ & $10,759(59.83 \%)$ \\
High expressed $(\geq 500)$ & $157(0.89 \%)$ & $6,668(38.20 \%)$ & $7,068(39.31 \%)$ \\
${ }^{1}$ RPKM = expected number of reads per kilobase of transcript sequence per million base pairs sequenced. \\
${ }^{2}$ CTR = control $($ GMEC treated with 0 $\mu M$ trans-10, cis-12 CLA); TR1 = GMEC treated with $100 \mu M$ trans- \\
10, cis-12 CLA; TR2 = GMEC treated with 200 $\mu$ M trans-10,cis-12 CLA).
\end{tabular}


better assess the molecular function of CLA in GMEC, we used Venn diagrams to show the common DEG in the 3 groups. We identified 48 common DEG of TR1

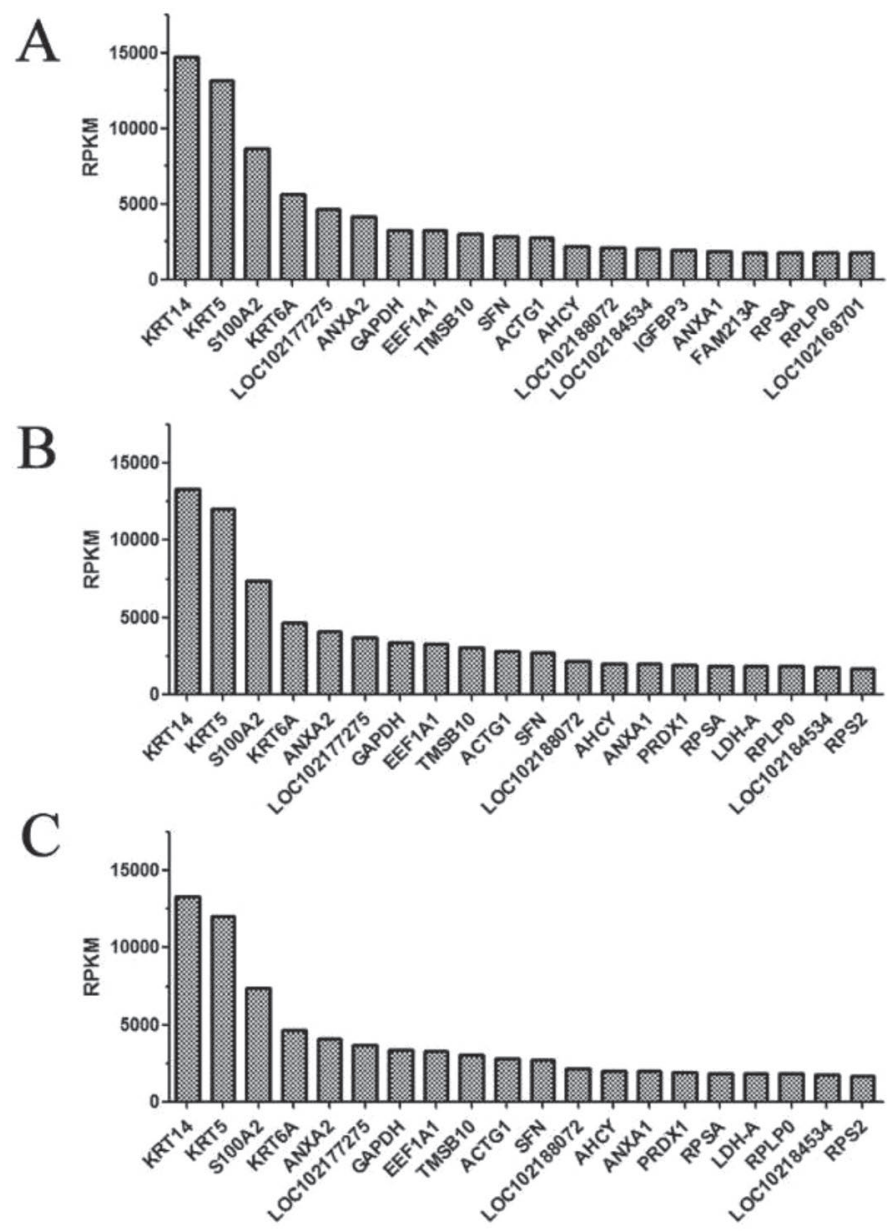

Figure 1. Top 20 highly expressed genes in different groups based on values for expected number of reads per kilobase of transcript sequence per million base pairs sequenced (RPKM): (A) CTR group [control; goat mammary epithelial cells (GMEC) treated with $0 \mu M$ trans-10, cis-12 CLA]; (B) TR1 group = GMEC treated with $100 \mu M$ trans-10,cis-12 CLA; (C) TR2 group = GMEC treated with $200 \mu M$ trans-10,cis-12 CLA. ACTG1 = actin gamma $1 ; A H C Y=$ adenosylhomocysteinase; $A N X A 1=$ annexin A1; $A N X A 2=$ annexin A2; EEF1A1 = eukaryotic translation elongation factor $1 \propto 1 ;$ FAM213A $=$ family with sequence similarity 213 member A; FTH1 = ferritin heavy chain 1; GAPDH = glyceraldehyde-3-phosphate dehydrogenase; $I G F B P 3=$ insulin-like growth factor binding protein $3 ; K R T 5=$ keratin $5 ; K R T 14=$ keratin $14 ; K R T 6 A=$ keratin $6 \mathrm{~A} ; L D H A=$ lactate dehydrogenase; LOC102168701 = uncharacterized LOC102168701; LOC102177275 = keratin, type I cytoskeletal 17; LOC102184534 = cystatin-C; LOC102188072 = metallothionein-2; PRDX1 = peroxiredoxin 1; RPL27A = ribosomal protein L27a; $R P S 2=$ ribosomal protein $\mathrm{S} 2 ; R P S 14=$ ribosomal protein $\mathrm{S} 14 ; R P L 35=$ ribosomal protein L35; $R P S A=$ ribosomal protein $\mathrm{SA} ; R P L P 0=$ ribosomal protein lateral stalk subunit P0; $S 100 A 10=\mathrm{S} 100$ calcium binding protein A10; $\mathrm{S} 100 \mathrm{~A} 14=\mathrm{S} 100$ calcium binding protein A14; S100A2 = S100 calcium binding protein $\mathrm{A} 2 ; S F N=$ stratifin; $T M S B 10=$ thymosin $\beta 10 ; U B B$ $=$ ubiquitin $\mathrm{B}$. versus CTR and TR2 versus CTR comparisons, indicating genes affected by any level of t10c12-CLA. Additionally, we identified 41 DEG shared by TR1 versus CTR, TR2 versus CTR, and TR1 versus TR2; the fold changes of these genes were dose-dependent (Figure 2, Supplemental Table S4; https://doi.org/10.3168/jds .2017-12822). To better understand the 41 common DEG, gene expression levels from RNA-seq results are listed in Supplemental Table S5 (https://doi.org/10 $.3168 /$ jds.2017-12822).

\section{Gene Ontology and Pathway Analysis of 41 DEGs}

The GO results based on the 41 common DEG are listed in Supplemental Table S6 (https://doi.org/10 $.3168 /$ jds.2017-12822). The most affected biological process was the monocarboxylic acid metabolic process, and the most affected molecular function was oxidoreductase activity, as shown in Figure 3. The top 20 pathways were then enriched (Figure 4 and Supplemental Table S7; https://doi.org/10.3168/jds.2017-12822). The affected pathways were almost all related to lipid metabolism (e.g., chx01212, chx00061, chx03320, and chx04152). Notably, fatty acid degradation and AMPK signaling pathway were affected greatly.

\section{Verification of DEG in GMEC in Response to CLA}

Real-time $\mathrm{qPCR}$ was used to validate selected differently expressed genes based on the RNA-seq results and the analysis. We tested 9 genes randomly from 41 DEG: acyl-CoA synthetase long-chain family member 4 (ACSL4), carnitine palmitoyltransferase $1 \mathrm{~A}$ $(\boldsymbol{C P T 1 A})$, fatty acid synthase $(\boldsymbol{F A S N})$, stearoyl-CoA desaturase 1 ( $\boldsymbol{S} \boldsymbol{C D} \mathbf{1})$, farnesyl diphosphate synthase $(\boldsymbol{F D P S})$, glucose-6-phosphate isomerase $(\boldsymbol{G P I}), 3$-hydroxy-3-methylglutaryl-CoA synthase 1 (HMGCS1), perilipin 2 (PLIN2), and solute carrier family 2 member 1 (SLC2A1). The correlations between the mRNA expression level from qPCR and RNA-seq were relatively high, with Pearson correlation coefficients of 0.9809 (TR1 vs. CTR, $P<0.0001$ ) and 0.9896 (TR1 vs. CTR, $P<0.0001$; Supplemental Figure S1; https:// doi.org/10.3168/jds.2017-12822), validating the reproducibility of gene expression data in this study. Meanwhile, the gene expression patterns demonstrated by RT-qPCR were similar to those from the RNA-seq results, as shown in Figure 5. Gene expression of $S C D 1$, $A C S L 4, F D P S, H M G C S 1, P L I N 2$, and SLC2A1 showed a significant dose-dependent effect with increasing concentrations of t10c12-CLA $(P<0.05$; Figure 5, panels $\mathrm{B}, \mathrm{C}, \mathrm{D}, \mathrm{E}, \mathrm{G}$, and $\mathrm{H})$. 


\section{T10c12-CLA Alters De Novo Fatty Acid Gene Expression Involved in Downregulating SREBP1 and Upregulating AMPK Signaling in GMEC}

The mRNA abundances of $A C A C A$ and SREBF1 were dramatically decreased in TR1 and TR2 $(P<$ 0.05 , Figure 5, panels $\mathrm{J}$ and $\mathrm{K}$ ). Protein expression of FASN and SCD1 was dramatically decreased in TR2 compared with CTR $(P<0.05$, Figure 6$)$. Activation of AMPK signaling was also detected in the treatment of t10c12-CLA. The phosphorylation of AMPK was increased with increasing concentration of t10c12-CLA, as was the phosphorylation of downstream enzyme ACACA, as shown in Figure 6.

\section{DISCUSSION}

Lipid metabolism is an important process in the mammary gland and can be regulated by nutritional factors, especially fatty acids (Peterson et al., 2004; Bernard et al., 2005). The effects of t10c12-CLA on milk fat content and composition are well established in several mammals (Hussein et al., 2013; Baldin et al., 2014; Harvatine et al., 2014). In addition to regulating milk fat, t10c12-CLA has been shown to reduce triglyceride storage, enhance fatty acid oxidation, and diminish glucose uptake and utilization in murine adipocytes (den Hartigh et al., 2013). Also, CLA alters body composition by regulating lipid metabolism in adipose tissue, as well as by modulating skeletal muscle metabolism (Kim et al., 2016). Because few studies have been performed with t10c12-CLA in small ruminants, the detailed mechanisms of t10c12-CLA on lipid metabolism in dairy goats are incompletely understood.

A previous study reported that only a high dose of t10c12-CLA provides an anti-lipogenic effect (Wang et al., 2014). To investigate this further, we used GMEC as the in vitro model in this study, and tested 0,10 , and $200 \mu M$ t10c12-CLA to evaluate whether the effects was dose-dependent. Then, RNA sequencing was performed to comprehensively evaluate the regulatory effects of t10c12-CLA in GMEC. From our data, 25,153 annotated transcripts were obtained after RNA sequencing and mapping to the reference genome. Different treatments shared the most highly expressed genes; for example, keratin 14 (KRT14), keratin 5 (KRT5), S100 calcium binding protein A2 (S100A2), and keratin $6 \mathrm{~A}$ $($ KRT6A). Keratins (including KRT14, KRT5, and $K R T 6 A)$ are intermediate filament-forming proteins that provide mechanical support and provide a variety of additional functions in epithelial cells (Schweizer et al., 2006). The S100 calcium binding proteins (such as S100A2) participate in a variety of intracellular and extracellular functions, affecting protein phosphorylation, transcription factors, $\mathrm{Ca}^{2+}$ homeostasis, the dynamics of cytoskeleton constituents, enzyme activities, cell growth and differentiation, and the inflammatory response (Donato, 2003). The ubiquitous expression mode indicates that these genes are necessary for cell function and metabolism.

We next investigated DEG by comparing the groups. We obtained 49, 854, and 623 DEG, respectively, when comparing CTR and TR1, CTR and TR2, and TR1 and TR2. We then focused on the common DEG, reasoning that these genes clearly exhibit significant dose-effect changes in response to t10c12-CLA. Genes involved in putative cellular signaling were enriched according to GO and KEGG pathway analysis. We found that the most affected GO and KEGG terms were closely related to fatty acid metabolism. Moreover, KEGG pathway analysis indicated that terpenoid backbone biosynthesis (chx00900) was enriched, with
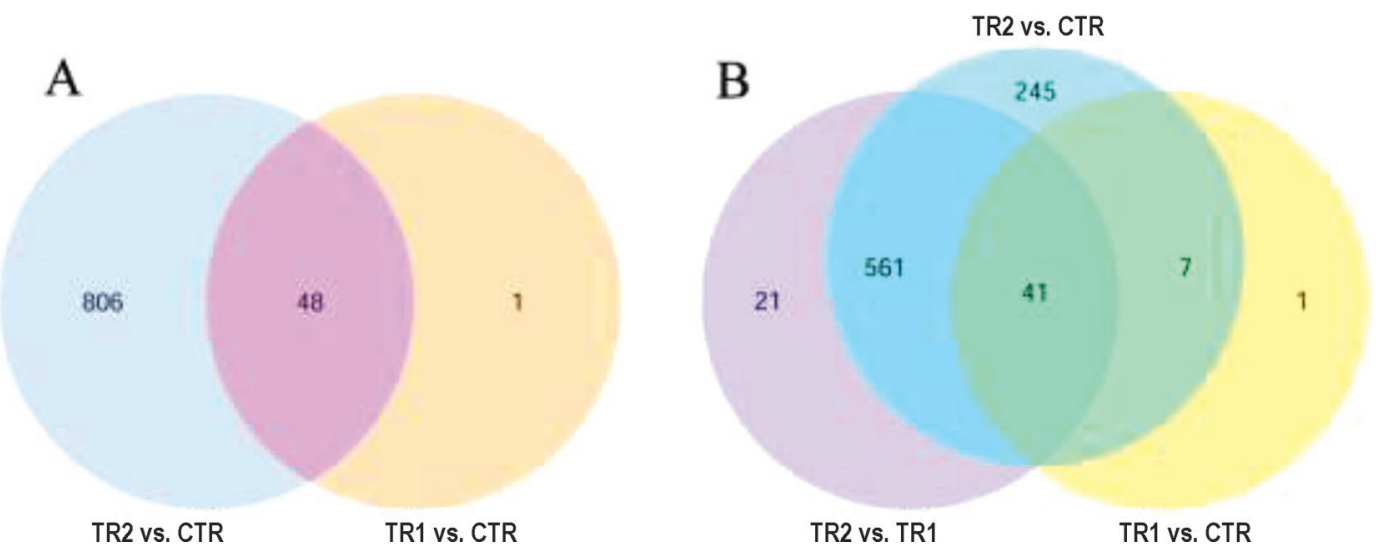

Figure 2. Venn diagram based on differentially expressed genes (DEG): (A) common DEG of TR1 (goat mammary epithelial cells, GMEC, treated with $100 \mu \mathrm{M}$ trans-10,cis-12 CLA) versus CTR (control; GMEC treated with $0 \mu M$ trans-10,cis-12 CLA) and TR2 (GMEC treated with $200 \mu \mathrm{M}$ trans-10,cis-12 CLA) versus CTR, regardless of dose response; (B) common DEG of TR1 versus CTR, TR2 versus CTR, and TR1 versus TR2, that exhibit dose-dependent changes. Color version available online. 
downregulation of FDPS and HMGCS1. The HMGCS1 enzyme converts acetyl CoA and acetoacetyl-CoA into 3-hydroxy-3-methylglutaryl CoA (HMG-CoA), then as the rate-limiting step, HMG-CoA reductase reduces HMG-CoA into mevalonate (Quintana et al., 2017). The FDPS enzyme catalyzes the formation of farnesyl diphosphate (FPP) by the head-to-tail condensation of dimethylallyl diphosphate with 2 molecules of isopentenyl diphosphate (Ericsson et al., 1996; Ishimoto et al., 2010). These findings indicated that t10c12-CLA might affect cellular cholesterol synthesis in GMEC. We also identified genes in adipocytokine signaling (KEGG ID: chx04920) and PPAR signaling (KEGG ID: chx03320) pathways, indicating that the effect of t10c12-CLA on

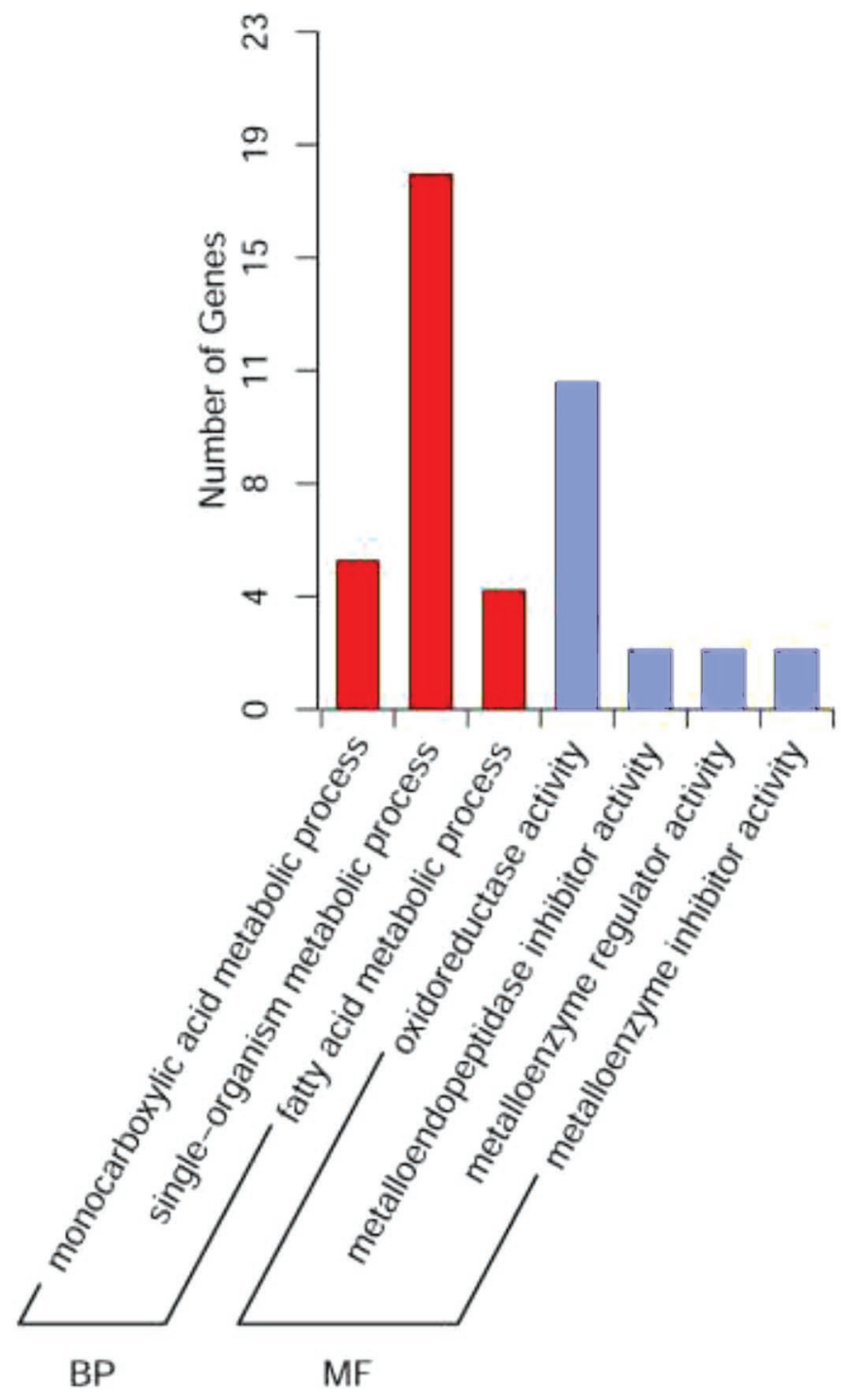

Figure 3. The significant Gene Ontology (GO) items of differentially expressed genes. The significance of GO was judged by a threshold of the corrected $P$-value $<0.05 . \mathrm{BP}=$ biological process; $\mathrm{MF}=$ molecular function. Color version available online.
GMEC might be correlated with PPAR $\gamma$ signaling and inflammation. Besides that, AMPK signaling pathway (KEGG ID: chx04152) was also involved.

To confirm the RNA-seq results, RT-qPCR was performed on 9 randomly selected DEG. The expression trends demonstrated by qPCR were similar to those from the RNA-seq results, and the comparison groups showed significant dose-dependent effects with increasing amount of t10c12-CLA. These results are in line with our expectations. Of the 41 DEG, FASN, SCD1, $A C S L 4$, and CPT1A were highly related to fatty acid metabolism.

Acetyl-CoA is the principal substrate of de novo fatty acid synthesis. In ruminants, acetate is produced in the rumen, transformed into pyruvate, and then further transformed into acetyl-CoA (Laliotis et al., 2010). The fatty acid synthesis pathway in the mammary gland involves 2 crucial enzymatic reactions. First, ACACA carboxylates acetyl-CoA to form malonyl-CoA, and then FASN utilizes both acetyl-CoA and malonyl-CoA to synthesize fatty acids (Chajès et al., 2006). The SREBP1 protein is commonly recognized as a pivotal transcription factor in mammary lipid metabolism (Bionaz and Loor, 2008). In its activated form, nuclear SREBP (nSREBP) is regulated by changes in the levels of oxysterols, insulin, glucose, and PUFA (Edwards et al., 2000). In hepatic lipid synthesis, AMP-activated kinase (AMPK), as a central regulator of cellular energy levels, is activated in response to the increase in the cellular AMP:ATP ratio. Activated AMPK inactivates ACACA by phosphorylation of this enzyme (Saggerson, 2008), resulting in suppression of fatty acid biosynthesis (Park et al., 2002). Previous study demonstrated that t10c12-CLA activates AMPK in a dose-dependent manner in rat skeletal muscle cells (Mohankumar et al., 2012). Docosahexaenoic acid, another PUFA, inhibits proteolytic processing of SREBP-1c via activation of AMPK (Deng et al., 2015). In the present study, 6 genes, including 4 DEG that regulate de novo fatty acid synthesis, triglyceride synthesis, and lipolysis (FASN, SCD1, ACSL4, and CPT1A), were verified using RT-qPCR. The findings of downregulation of FASN, $A C A C A$, and $S C D 1$ with an increasing concentration of t10c12-CLA were similar with those in bovine mammary epithelial cells (Kadegowda et al., 2009; Wang et al., 2014).

The protein levels of FASN and SCD1 and phosphorylation of AMPK and ACACA were detected by Western blot. The results indicated that t10c12-CLA possibly activated AMPK signaling and then increased the inhibition of ACACA activity in GMEC. Activation of AMPK also results in the activation of carnitine palmitoyltransferase 1 (CPT1; Kim et al., 2016), which acts as a rate-limiting enzyme and plays a pivotal 
part in regulation of long-chain fatty acid oxidation in mitochondria (New et al., 1998) by catalyzing longchain fatty acid translocation into the mitochondrial matrix (Louet et al., 2001). Supplementation of CLA in mice upregulates CPT1 in skeletal muscle, suggesting increased energy expenditure and fatty acid oxidation (Park and Park, 2012). In the present study, upregulation of CPT1A was identified by RNA-seq and verified by RT-qPCR. A previous study on GMEC showed that AMPK activation increased CPT1 mRNA level and decreased FASN and ACACA mRNA levels (Zhang et al., 2011). Based on these findings, we conclude that t10c12-CLA may regulate lipid metabolism through AMPK signaling, accompanied by the downregulation of de novo fatty acid synthesis in GMEC. However, a study on murine mammary gene expression in the lactation period indicated that during active lactation, genes involved in fatty acid $\beta$-oxidation are largely downregulated, and genes involves in lipogenesis are upregulated, driving lipid substrates to be utilized for milk fat synthesis (Rudolph et al., 2007). Fatty acid synthesis, rather than oxidation, predominates in the mammary gland during lactation (Rawson et al., 2012). Although we found upregulation of $C P T 1 A$ in response to t10c12-CLA, whether $\beta$-oxidation is upregulated in goat mammary gland remains to be investigated basing on the specific physiological environment.

\section{CONCLUSIONS}

This study greatly expanded our understanding and provided additional insight into the role of CLA on milk lipid metabolism in dairy goats. Through RNAsequencing, critical genes and pathways were identified. Overall, t10c12-CLA inhibits fatty acid synthesis through downregulation of genes involved in de novo fatty acid synthesis in GMEC, and this process is likely to correlate with activation of the AMPK signaling pathway.

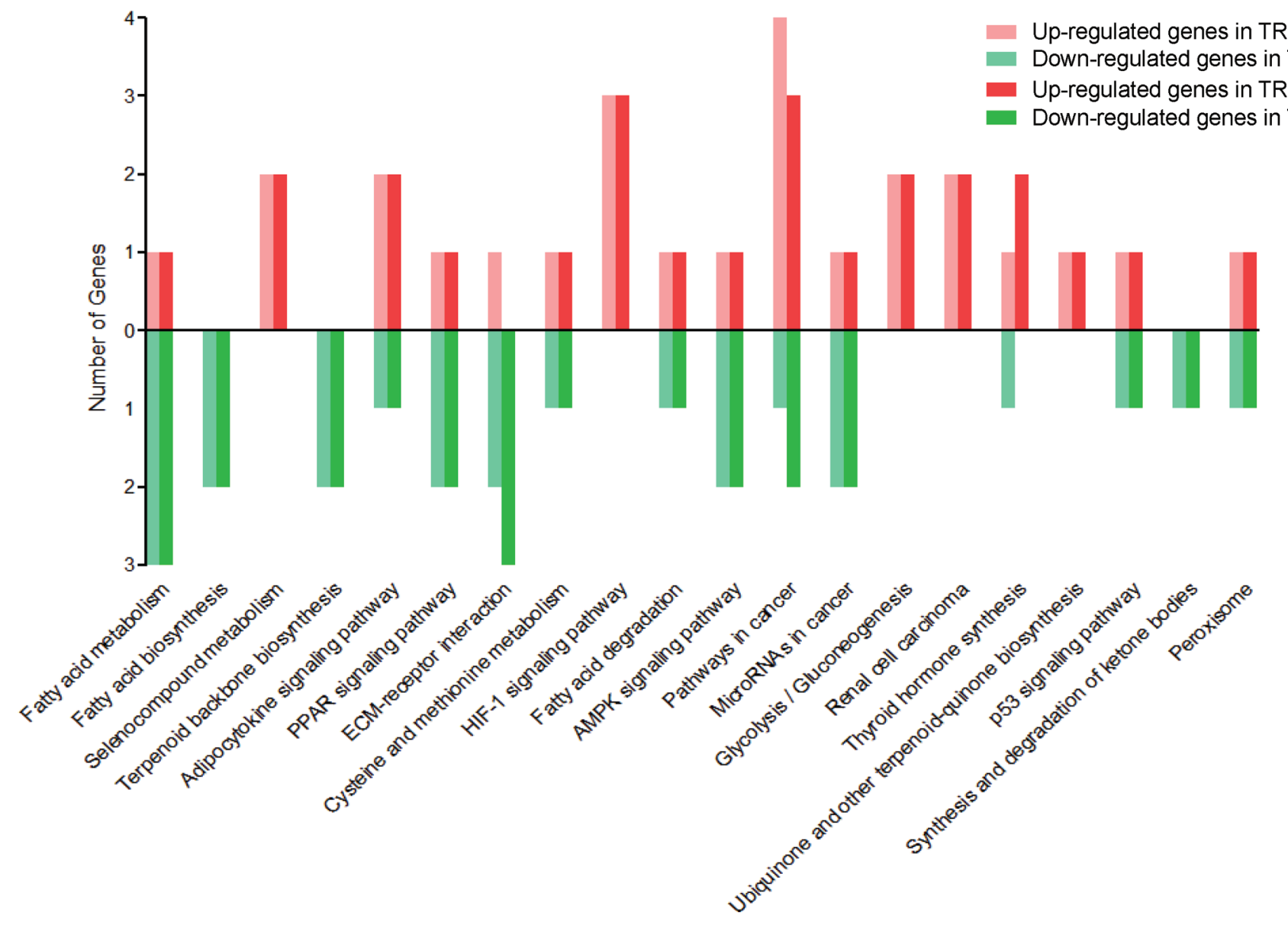

Figure 4. Pathways enriched by Kyoto Encyclopedia of Genes and Genomes (KEGG) of differentially expressed genes. Top 20 KEGG pathways were listed according to the significantly enriched items based on corrected $P$-value. CTR $=$ control; goat mammary epithelial cells (GMEC) treated with $0 \mu M$ trans-10,cis-12 CLA; TR1 = GMEC treated with $100 \mu M$ trans-10, cis-12 CLA; TR2 = GMEC treated with $200 \mu M$ trans-10, cis-12 CLA. Color version available online. 


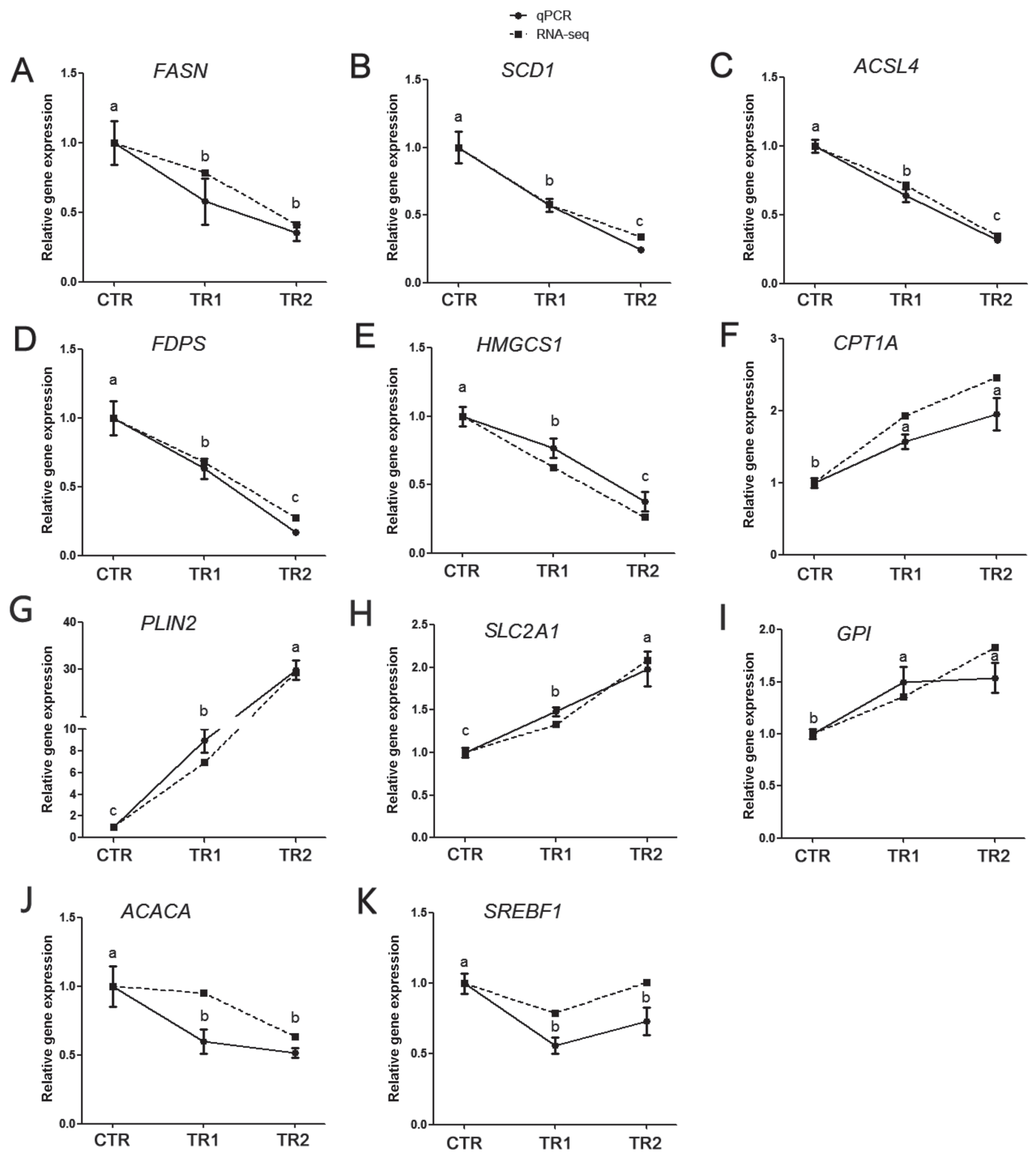

Figure 5. Verification of gene expressions by quantitative (q)PCR. For RNA-seq results (----), the relative gene expression levels were calculated as the fold change. For the real-time-qPCR results $(--)$, the relative gene expression levels were calculated using the $2^{-\Delta \Delta} \mathrm{Ct}(\mathrm{cycle}$ threshold) method with UXT, RPS9, and MRPL39 as internal control genes. CTR = control; goat mammary epithelial cells (GMEC) treated with $0 \mu M$ trans-10,cis-12 CLA; TR1 = GMEC treated with $100 \mu M$ trans-10,cis-12 CLA; TR2 = GMEC treated with $200 \mu M$ trans-10,cis-12 CLA. Different letters $(\mathrm{a}, \mathrm{b}, \mathrm{c})$ indicate significant differences in qPCR results at $P<0.05$. Error bars indicate SE 


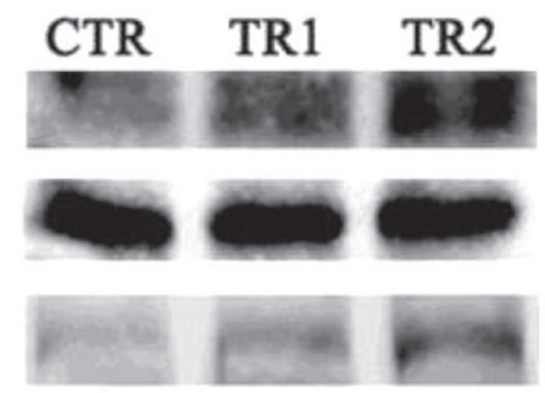

$\mathrm{p}-\mathrm{AMPK} \alpha$
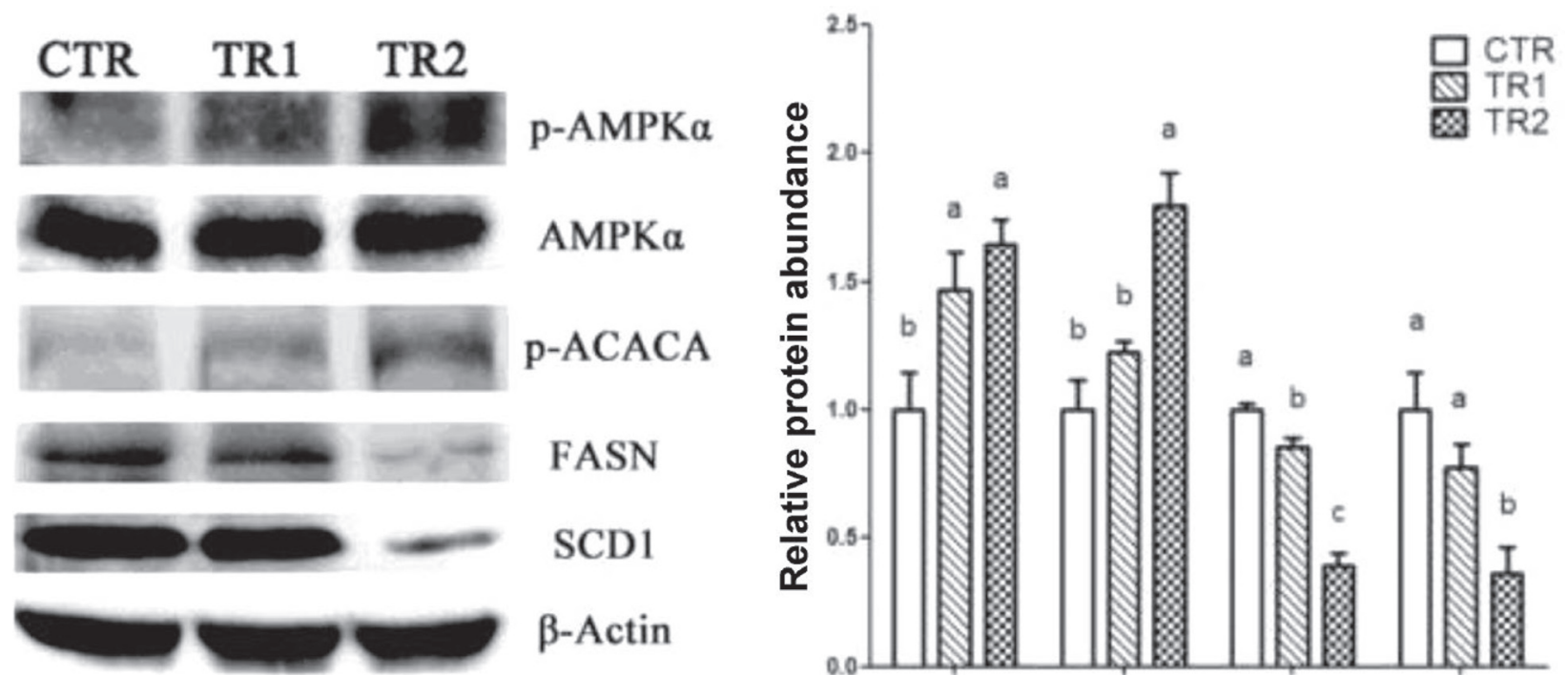

AMPKa

p-ACACA

FASN

SCD1

$\beta$-Actin

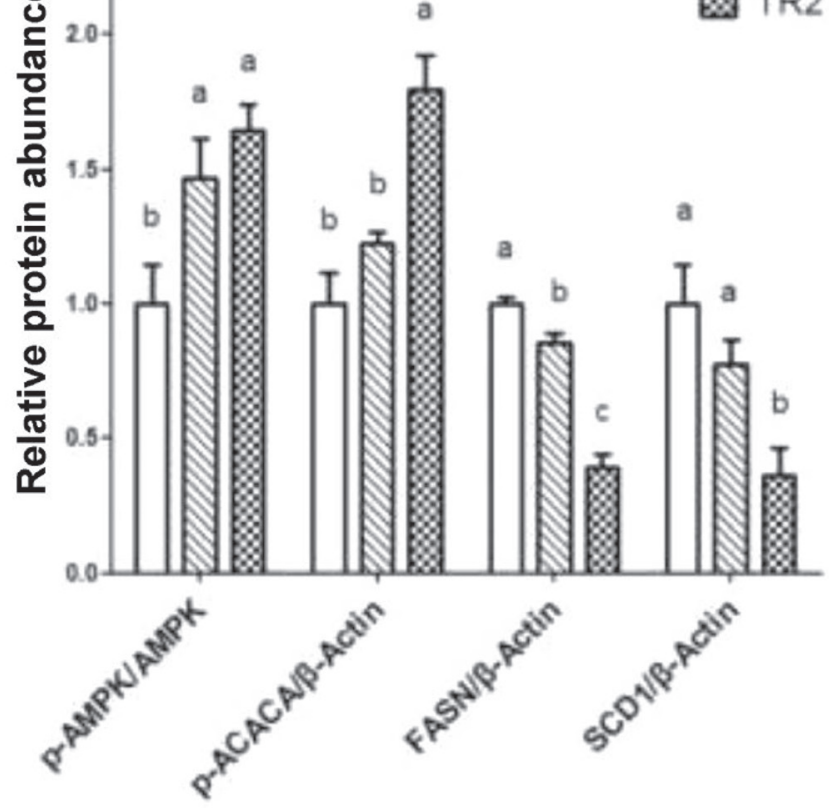

Figure 6. Verification of protein abundances of fatty acid synthase (FASN), stearoyl-CoA desaturase 1 (SCD1), and AMP-activated protein kinase (AMPK) signaling pathway. The phosphorylation level of AMPK was normalized to AMPK and the protein abundance of phosphorylated (p)-ACACA (acetyl-CoA carboxylase 1), FASN, and SCD1 was normalized to $\beta$-actin. The blots are representative of 3 independent experiments with similar results. CTR = control; goat mammary epithelial cells (GMEC) treated with $0 \mu M$ trans-10, cis-12 CLA; TR1 = GMEC treated with $100 \mu M$ trans-10,cis-12 CLA; TR2 = GMEC treated with $200 \mu M$ trans-10,cis-12 CLA. Different letters (a, b, c) indicate significant differences in results at $P<0.05$. Error bars indicate SE.

\section{ACKNOWLEDGMENTS}

This research was jointly supported by the National Natural Science Foundation of China (Beijing; 31272409 and 31672398) and the Science Foundation of Shaanxi province of China (Xi'an, 2016KTZDNY02-05).

\section{REFERENCES}

Baldin, M., R. Dresch, J. Souza, D. Fernandes, M. A. S. Gama, K. J. Harvatine, and D. E. Oliveira. 2014. CLA induced milk fat depression reduced dry matter intake and improved energy balance in dairy goats. Small Rumin. Res. 116:44-50.

Baumgard, L. H., B. A. Corl, D. A. Dwyer, A. Saebø, and D. E. Bauman. 2000. Identification of the conjugated linoleic acid isomer that inhibits milk fat synthesis. Am. J. Physiol. Regul. Integr. Comp. Physiol. 278:R179-R184.

Baumgard, L. H., E. Matitashvili, B. A. Corl, D. A. Dwyer, and D. E. Bauman. 2002. trans-10, cis-12 conjugated linoleic acid decreases lipogenic rates and expression of genes involved in milk lipid synthesis in dairy cows. J. Dairy Sci. 85:2155-2163.

Benjamini, Y., and Y. Hochberg. 1995. Controlling the false discovery rate: A practical and powerful approach to multiple testing. J.R. Stat. Soc. 57:289-300.

Bernard, L., J. Rouel, C. Leroux, A. Ferlay, Y. Faulconnier, P. Legrand, and Y. Chilliard. 2005. Mammary lipid metabolism and milk fatty acid secretion in alpine goats fed vegetable lipids. J. Dairy Sci. 88:1478-1489.

Bionaz, M., and J. J. Loor. 2008. Gene networks driving bovine milk fat synthesis during the lactation cycle. BMC Genomics 9:366.
Byron, S. A., K. R. Van Keuren-Jensen, D. M. Engelthaler, J. D. Carpten, and D. W. Craig. 2016. Translating RNA sequencing into clinical diagnostics: opportunities and challenges. Nat. Rev. Genet. 17:257-271.

Chajès, V., M. Cambot, K. Moreau, G. M. Lenoir, and V. Joulin. 2006. Acetyl-CoA carboxylase alpha is essential to breast cancer cell survival. Cancer Res. 66:5287-5294.

Chilliard, Y., F. Glasser, A. Ferlay, L. Bernard, J. Rouel, and M. Doreau. 2007. Diet, rumen biohydrogenation and nutritional quality of cow and goat milk fat. Eur. J. Lipid Sci. Technol. 109:828855.

den Hartigh, L. J., C. Y. Han, S. Wang, M. Omer, and A. Chait. 2013. 10E,12Z-conjugated linoleic acid impairs adipocyte triglyceride storage by enhancing fatty acid oxidation, lipolysis, and mitochondrial reactive oxygen species. J. Lipid Res. 54:2964-2978.

Deng, X., Q. Dong, D. Bridges, R. Raghow, E. A. Park, and M. B Elam. 2015. Docosahexaenoic acid inhibits proteolytic processing of sterol regulatory element-binding protein-1c (SREBP-1c) via activation of AMP-activated kinase. Biochim. Biophys. Acta 1851:1521-1529.

Donato, R. 2003. Intracellular and extracellular roles of S100 proteins. Microsc. Res. Tech. 60:540-551.

Edwards, P. A., D. Tabor, H. R. Kast, and A. Venkateswaran. 2000. Regulation of gene expression by SREBP and SCAP. Biochim. Biophys. Acta 1529:103-113.

Ericsson, J., S. M. Jackson, B. C. Lee, and P. A. Edwards. 1996. Sterol regulatory element binding protein binds to a cis element in the promoter of the farnesyl diphosphate synthase gene. Proc. Natl. Acad. Sci. USA 93:945-950.

Harvatine, K. J., and D. E. Bauman. 2006. SREBP1 and thyroid hormone responsive spot 14 (S14) are involved in the regulation of bovine mammary lipid synthesis during diet-induced milk fat depression and treatment with CLA. J. Nutr. 136:2468-2474. 
Harvatine, K. J., M. M. Robblee, S. R. Thorn, Y. R. Boisclair, and D. E. Bauman. 2014. Trans-10, cis-12 CLA dose-dependently inhibits milk fat synthesis without disruption of lactation in C57BL/6J mice. J. Nutr. 144:1928-1934.

Hussein, M., K. H. Harvatine, W. M. Weerasinghe, L. A. Sinclair, and D. E. Bauman. 2013. Conjugated linoleic acid-induced milk fat depression in lactating ewes is accompanied by reduced expression of mammary genes involved in lipid synthesis. J. Dairy Sci. 96:3825-3834.

Ishimoto, K., K. Tachibana, I. Hanano, D. Yamasaki, H. Nakamura, M. Kawai, Y. Urano, T. Tanaka, T. Hamakubo, J. Sakai, T. Kodama, and T. Doi. 2010. Sterol-regulatory-element-binding protein 2 and nuclear factor Y control human farnesyl diphosphate synthase expression and affect cell proliferation in hepatoblastoma cells. Biochem. J. 429:347-357.

Jakhesara, S. J., P. G. Koringa, V. D. Bhatt, T. M. Shah, S. Vangipuram, S. Shah, and C. G. Joshi. 2013. RNA-Seq reveals differentially expressed isoforms and novel splice variants in buccal mucosal cancer. Gene 516:24-32.

Kadegowda, A. K., M. Bionaz, L. S. Piperova, R. A. Erdman, and J. J. Loor. 2009. Peroxisome proliferator-activated receptor-gamma activation and long-chain fatty acids alter lipogenic gene networks in bovine mammary epithelial cells to various extents. J. Dairy Sci. 92:4276-4289.

Kadegowda, A. K., E. E. Connor, B. B. Teter, J. Sampugna, P. Delmonte, L. S. Piperova, and R. A. Erdman. 2010. Dietary trans fatty acid isomers differ in their effects on mammary lipid metabolism as well as lipogenic gene expression in lactating mice. J. Nutr. 140:919-924.

Kadegowda, A. K., M. J. Khan, L. S. Piperova, B. B. Teter, S. L. Rodriguez-Zas, R. A. Erdman, and J. J. Loor. 2013. Trans-10, cis12-conjugated linoleic acid-induced milk fat depression is associated with inhibition of PPARgamma signaling and inflammation in murine mammary tissue. J. Lipids 2013:890343.

Kim, Y., J. Kim, K. Y. Whang, and Y. Park. 2016. Impact of conjugated linoleic acid (CLA) on skeletal muscle metabolism. Lipids $51: 159-178$.

Laliotis, G. P., I. Bizelis, and E. Rogdakis. 2010. Comparative approach of the de novo fatty acid synthesis (lipogenesis) between ruminant and non ruminant mammalian species: From biochemical level to the main regulatory lipogenic genes. Curr. Genomics 11:168-183.

Langmead, B., and S. L. Salzberg. 2012. Fast gapped-read alignment with Bowtie 2. Nat. Methods 9:357-359.

Louet, J. F., C. Le May, J. P. Pégorier, J. F. Decaux, and J. Girard. 2001. Regulation of liver carnitine palmitoyltransferase I gene expression by hormones and fatty acids. Biochem. Soc. Trans. 29:310-316.

Mohankumar, S. K., C. G. Taylor, L. Siemens, and P. Zahradka. 2012. Acute exposure of L6 myotubes to cis-9, trans-11 and trans-10, cis-12 conjugated linoleic acid isomers stimulates glucose uptake by modulating $\mathrm{Ca}^{2+} /$ calmodulin-dependent protein kinase II. Int. J. Biochem. Cell Biol. 44:1321-1330.

Mortazavi, A., B. A. Williams, K. McCue, L. Schaeffer, and B. Wold. 2008. Mapping and quantifying mammalian transcriptomes by RNA-Seq. Nat. Methods 5:621-628.

Nakamura, M. T., Y. Cheon, Y. Li, and T. Y. Nara. 2004. Mechanisms of regulation of gene expression by fatty acids. Lipids 39:10771083.

New, K. J., K. R. F. Elliott, and P. A. Quant. 1998. Flux control exerted by mitochondrial outer membrane carnitine palmitoyltransferase over ketogenic flux in hepatocytes isolated from suckling rats. Biochem. Soc. Trans. 26:S88.

Park, S. H., S. R. Gammon, J. D. Knippers, S. R. Paulsen, D. S. Rubink, and W. W. Winder. 2002. Phosphorylation-activity relationships of AMPK and acetyl-CoA carboxylase in muscle. J. Appl. Physiol. 92:2475-2482.

Park, Y., and Y. Park. 2012. Conjugated fatty acids increase energy expenditure in part by increasing voluntary movement in mice. Food Chem. 133:400-409.
Peterson, D. G., E. A. Matitashvili, and D. E. Bauman. 2004. The inhibitory effect of trans-10, cis-12 CLA on lipid synthesis in bovine mammary epithelial cells involves reduced proteolytic activation of the transcription factor SREBP-1. J. Nutr. 134:2523-2527.

Quintana, A. M., J. A. Hernandez, and C. G. Gonzalez. 2017. Functional analysis of the zebrafish ortholog of HMGCS1 reveals independent functions for cholesterol and isoprenoids in craniofacial development. PLoS One 12:e0180856.

Rawson, P., C. Stockum, L. Peng, B. Manivannan, K. Lehnert, H. E Ward, S. D. Berry, S. R. Davis, R. G. Snell, D. McLauchlan, and T. W. Jordan. 2012. Metabolic proteomics of the liver and mammary gland during lactation. J. Proteomics 75:4429-4435.

Robinson, M. D. 2010. edgeR: A Bioconductor package for differential expression analysis of digital gene expression data. Bioinformatics 26:139-140.

Robinson, M. D., and A. Oshlack. 2010. A scaling normalization method for differential expression analysis of RNA-seq data. Genome Biol. 11:R25.

Rudolph, M. C., M. C. Neville, and S. M. Anderson. 2007. Lipid synthesis in lactation: Diet and the fatty acid switch. J. Mammary Gland Biol. Neoplasia 12:269-281.

Saggerson, D. 2008. Malonyl-CoA, a key signaling molecule in mammalian cells. Annu. Rev. Nutr. 28:253-272.

Sajnani, M. R., A. K. Patel, V. D. Bhatt, A. K. Tripathi, V. B. Ahir, V. Shankar, S. Shah, T. M. Shah, P. G. Koringa, S. J. Jakhesara, and C. G. Joshi. 2012. Identification of novel transcripts deregulated in buccal cancer by RNA-seq. Gene 507:152-158.

Schweizer, J., P. E. Bowden, P. A. Coulombe, L. Langbein, E. B. Lane, T. M. Magin, L. Maltais, M. B. Omary, D. A. Parry, M. A. Rogers, and M. W. Wright. 2006. New consensus nomenclature for mammalian keratins. J. Cell Biol. 174:169-174.

Somasundaram, I., R. Mishra, H. Radhakrishnan, R. Sankaran, V. N. Garikipati, and D. Marappagounder. 2015. Human adult stem cells maintain a constant phenotype profile irrespective of their origin, basal media, and long term cultures. Stem Cells Int. 2015:146051.

Trapnell, C., L. Pachter, and S. L. Salzberg. 2009. TopHat: Discovering splice junctions with RNA-Seq. Bioinformatics 25:1105-1111.

Vandesompele, J., K. De Preter, F. Pattyn, B. Poppe, N. Van Roy, A. De Paepe, and F. Speleman. 2002. Accurate normalization of real-time quantitative RT-PCR data by geometric averaging of multiple internal control genes. Genome Biol. 3:RESEARCH0034.

Wang, H., H. Liu, J. Liu, K. Zhao, C. Wang, and W. Yang. 2014 High-level exogenoustrans10,cis12 conjugated linoleic acid plays an anti-lipogenesis role in bovine mammary epithelial cells. Anim. Sci. J. 85:744-750.

Wang, H., J. Luo, Z. Chen, W. T. Cao, H. F. Xu, D. M. Gou, and J. J. Zhu. 2015. MicroRNA-24 can control triacylglycerol synthesis in goat mammary epithelial cells by targeting the fatty acid synthase gene. J. Dairy Sci. 98:9001-9014.

Wang, L., Z. Feng, X. Wang, X. Wang, and X. Zhang. 2010. DEGseq: An $R$ package for identifying differentially expressed genes from RNA-seq data. Bioinformatics 26:136-138.

Xie, C., X. Mao, J. Huang, Y. Ding, J. Wu, S. Dong, L. Kong, G. Gao, C. Y. Li, and L. Wei. 2011. KOBAS 2.0: A web server for annotation and identification of enriched pathways and diseases. Nuc. Acids Res. 39(Web Server issue):W316-322.

Xu, H. F., J. Luo, W. S. Zhao, Y. C. Yang, H. B. Tian, H. B. Shi, and M. Bionaz. 2016. Overexpression of SREBP1 (sterol regulatory element binding protein 1) promotes de novo fatty acid synthesis and triacylglycerol accumulation in goat mammary epithelial cells. J. Dairy Sci. 99:783-795.

Young, M. D., M. J. Wakefield, G. K. Smyth, and A. Oshlack. 2010. Gene ontology analysis for RNA-seq: Accounting for selection bias. Genome Biol. 11:R14.

Zhang, N., Q. Z. Li, X. J. Gao, and H. B. Yan. 2011. Potential role of adenosine monophosphate-activated protein kinase in regulation of energy metabolism in dairy goat mammary epithelial cells. J. Dairy Sci. 94:218-222. 